\title{
UNIVERSALITY LIMITS FOR ENTIRE FUNCTIONS
}

\author{
MISHKO MITKOVSKI
}

(Communicated by Richard Rochberg)

\begin{abstract}
Various statements on the distribution of eigenvalues of random matrices are obtained by considering the limiting behavior of the reproducing kernels of a certain naturally associated sequence of orthogonal polynomials. We establish a universal limiting behavior of this type in the case when the underlying measure does not have finite moments. In this case the orthogonal polynomials are replaced by a nested family of de Branges spaces of entire functions.
\end{abstract}

\section{INTRODUCTION AND MAIN RESULT}

Universality results in random matrix theory say that the local eigenvalue statistics of many random matrix ensembles do not depend on the exact probability distribution that is put on a set of matrices, but only on some general characteristics of the ensemble. Mathematically, most of these results are due to the universal limiting behavior of the Christoffel-Darboux reproducing kernels, which are independent of the underlying measure from which they arise. In the classical Gaussian case, this limiting behavior is found using the known, classical asymptotics of the Hermite polynomials. Establishing the asymptotic behavior of the orthogonal polynomials coming from a more general, non-Gaussian weight is usually a difficult task. This was first done by Pastur and Scherbina [8] for certain classes of weights. Very soon afterwards a powerful Riemann-Hilbert steepest descent method [3] was applied by Deift et al. [1,2 to cover even more classes of non-Gaussian weights.

More recently it was observed by Lubinsky [ 6 , that the universal behavior of the scaling limit can be established more directly without knowing the asymptotics of the corresponding orthogonal polynomials. He considered a simplified case, but one that already contains all the difficulties in the original limit. Namely, he showed that for a given regular weight $d \mu(x)=w(x) d x+d \mu_{s}(x)$ supported on $[-1,1]$ (a simple sufficient condition for this kind of regularity is that $w(x)>0$ a.e. on the support), the corresponding Christoffel-Darboux reproducing kernels $K_{N}(x, y)$ satisfy the following universal limiting behavior. For any $\xi_{0}$ in the interior of the absolutely continuous spectrum of $\mu$ with $w(x)$ positive and continuous at $\xi_{0}$ and for any $a, b \in \mathbb{R}$,

$$
\lim _{n \rightarrow \infty} \frac{K_{N}\left(\xi_{0}+\frac{a}{N}, \xi_{0}+\frac{b}{N}\right)}{K_{N}\left(\xi_{0}, \xi_{0}\right)}=\frac{\sin \rho\left(\xi_{0}\right)(a-b)}{\rho\left(\xi_{0}\right)(a-b)},
$$

Received by the editors November 14, 2011.

2010 Mathematics Subject Classification. Primary 30D20.

Key words and phrases. Universality limits, entire functions, reproducing kernels.

The author was supported in part by NSF grants \#DMS-1001098 and \#DMS-1101251.

(C) 2013 American Mathematical Society 
where $\rho(x)=1 /\left(\pi \sqrt{1-x^{2}}\right)$. This limiting behavior is universal in the sense that the limit is largely independent of the measure $\mu$. Many extensions and generalizations of this result were subsequently given by Levin [5], Totik [10], Simon [9], and Lubinsky [7] himself.

The purpose of this short note is to show that a similar limiting behavior can be established even for measures $\mu$ with infinite moments. Note that such measures do not produce orthogonal polynomials, and hence in this situation ChristoffelDarboux reproducing kernels $K_{N}(x, y)$ cannot even be defined.

In place of polynomials, for every $T>0$ we consider the space $\mathcal{E}_{T}(\mu)$ of all entire functions of exponential type $\leq T$ which belong in $L^{2}(\mu)$. We assume that $d \mu$ is a Poisson summable measure, i.e., $\int d \mu(t) /\left(1+t^{2}\right)<\infty$. For such measures $\mathcal{E}_{T}(\mu)$ is nontrivial for every $T>0$. It is a well-known theorem of Krein [4 that $\mathcal{E}_{T}(\mu)$ is either a closed proper subspace of $L^{2}(\mu)$ or it is dense in $L^{2}(\mu)$. One way to tell when the positive alternative holds is through the following majorant:

$$
m_{T}(w)=\sup \left\{|F(w)|: F \in \mathcal{E}_{T}(\mu),\|F\|_{L^{2}(\mu)} \leq 1\right\} ;
$$

namely, $\mathcal{E}_{T}(\mu)$ is not dense in $L^{2}(\mu)$ if $\log m_{T}(t)$ is a Poisson integrable function on the real line. It is not difficult to see that this is true for all $T>0$ if $\mu$ is a regular measure in the following sense.

Definition 1.1. We say that a measure $d \mu$ is regular if for any $\epsilon>0$ and any $t$ in the support of $\mu$ the following inequality holds:

$$
m_{T}(t) \leq C e^{\epsilon T},
$$

with $C$ independent of $t$ and $T$.

For example, any $d \mu(t)=w(t) d t$ which is Poisson integrable with $w(t) \geq c>0$ a.e. on $\mathbb{R}$, is regular.

In the case of a regular $\mu$, each $\mathcal{E}_{T}(\mu)$ has the structure of a de Branges space, and therefore for all $T>0$ the reproducing kernels $K_{T}(z, w)$ exist. These will play the role of the Christoffel-Darboux kernels in our analog of (1.1). Notice that $m_{T}(w)=\sqrt{K_{T}(w, w)}$. The fact that $\mu$ is Poisson integrable implies that this de Branges space is always regular and hence contains only entire functions from the Cartwright class.

For regular measures we will prove the following analog of (1.1).

Theorem 1.2. Let $\mu$ be a Poisson summable positive Borel measure on $\mathbb{R}$ that is regular. Assume that $\mu$ is absolutely continuous in an open set containing a point $\xi_{0}$ and that the density $w(t)$ is positive and continuous at $\xi_{0}$. Then for all $a, b \in \mathbb{R}$ the following equality holds:

$$
\lim _{T \rightarrow \infty} \frac{K_{T}\left(\xi_{0}+a / T, \xi_{0}+b / T\right)}{K_{T}\left(\xi_{0}, \xi_{0}\right)}=\frac{\sin (a-b)}{a-b} .
$$

Notice that this limit makes sense even in the case $a=b$, in which case the right hand side is just 1 .

The simplest example is obtained in the case when $d \mu(t)=d m(t)$ is the Lebesgue measure. In this case clearly $\mathcal{E}_{T}(m)=\mathrm{PW}_{T}$ is the usual Paley-Wiener space. For the reproducing kernels

$$
S_{T}(z, w)=\frac{\sin T(z-\bar{w})}{\pi(z-\bar{w})}
$$


in the Paley-Wiener space we have that

$$
\frac{S_{T}\left(\xi_{0}+a / T, \xi_{0}+b / T\right)}{S_{T}\left(\xi_{0}, \xi_{0}\right)}=\frac{\sin (a-b)}{(a-b)},
$$

and therefore in this case Theorem 1.2 trivially holds.

\section{Proof of Theorem 1.2}

Our strategy will be very similar to the one in $[6$. We will first prove the special case when $a=b$.

Proposition 2.1. Let $\mu$ be a Poisson summable positive Borel measure on $\mathbb{R}$ that is regular. Assume that $\mu$ is absolutely continuous in an open set containing a point $\xi_{0}$ and that the density $w(t)$ is positive and continuous at $\xi_{0}$. Let $a>0$. Then

$$
\lim _{T \rightarrow \infty} \frac{K_{T}\left(\xi_{0}+a / T, \xi_{0}+a / T\right)}{K_{T}\left(\xi_{0}, \xi_{0}\right)}=1 .
$$

Proof. We will prove the following stronger statement:

$$
\lim _{T \rightarrow \infty} \frac{K_{T}\left(\xi_{0}+a / T, \xi_{0}+a / T\right)}{T}=\frac{1}{\pi w\left(\xi_{0}\right)} .
$$

Let $\epsilon>0$ and choose $\delta>0$ small enough so that $\mu$ is absolutely continuous on $\left(\xi_{0}-\delta, \xi_{0}+\delta\right)$ and such that

$$
\frac{1}{1+\epsilon} \leq \frac{w\left(\xi_{0}\right)}{w(t)} \leq 1+\epsilon
$$

for all $t \in\left(\xi_{0}-\delta, \xi_{0}+\delta\right)$. Define a measure $\mu^{*}$ which is equal to $\mu$ everywhere outside $\left(\xi_{0}-\delta, \xi_{0}+\delta\right)$ and is absolutely continuous on $\left(\xi_{0}-\delta, \xi_{0}+\delta\right)$ with density $w^{*}(t)$ satisfying

$$
w^{*}(t)=w\left(\xi_{0}\right)(1+\epsilon), \quad t \in\left(\xi_{0}-\delta, \xi_{0}+\delta\right) .
$$

For this measure we clearly have $\mu \leq \mu^{*}$, and therefore $K_{T}^{*}(t, t) \leq K_{T}(t, t)$ for all $t \in \mathbb{R}$. We now find an upper bound of $1 / K_{T}^{*}(t, t)$ on the interval $t \in\left(\xi_{0}-\delta, \xi_{0}+\delta\right)$. Fix $\xi \in\left(\xi_{0}-\delta / 2, \xi_{0}+\delta / 2\right)$. Define

$$
G(z)=G_{\xi, \delta}(z)=\frac{\sin 4(z-\xi) / \delta}{4(z-\xi) / \delta} .
$$

Clearly, $G(\xi)=1$ and $|G(t)| \leq 1 / 2<1$, for $t \notin\left(\xi_{0}-\delta, \xi_{0}+\delta\right)$. In addition, $G(z)$ is entire of exponential type $4 / \delta$ and is bounded on the real line by 1 . Let $\eta \in\left(0, \frac{1}{2}\right)$ be arbitrary. For a given $T>0$ define

$$
\tau(T)=T-\frac{4}{\delta}\left\lfloor\frac{\eta \delta T}{4}\right\rfloor(\geq(1-\eta) T)
$$

Now, let

$$
F(z)=\frac{\sin \tau(z-\xi)}{\tau(z-\xi)}
$$

Clearly, $F(\xi)=1,\|F\|_{L^{2}(m)}=\sqrt{\frac{\pi}{\tau}}$, and $F(z)$ is bounded by 1 on the whole real line. Finally, for $T$ so large that $\left\lfloor\frac{\eta \delta T}{4}\right\rfloor \geq 1$, define

$$
H(z)=F(z) G(z)^{\left\lfloor\frac{\eta \delta T}{4}\right\rfloor} .
$$


The exponential type of $H(z)$ is less than or equal to $\tau+\frac{4}{\delta}\left\lfloor\frac{\eta \delta T}{4}\right\rfloor=T$ and $H(\xi)=1$.

Therefore, for all $\xi \in\left(\xi_{0}-\delta / 2, \xi_{0}+\delta / 2\right)$ we have

$$
\begin{aligned}
\frac{1}{K_{T}(\xi, \xi)} \leq & \frac{1}{K_{T}^{*}(\xi, \xi)} \leq \int_{\xi_{0}-\delta}^{\xi_{0}+\delta}|H(t)|^{2} d \mu^{*}(t)+\int_{\mathbb{R} \backslash\left(\xi_{0}-\delta, \xi_{0}+\delta\right)}|H(t)|^{2} d \mu^{*}(t) \\
\leq & w\left(\xi_{0}\right)(1+\epsilon) \int_{\xi_{0}-\delta}^{\xi_{0}+\delta}|H(t)|^{2} d t \\
& +\sup _{t \notin\left(\xi_{0}-\delta, \xi_{0}+\delta\right)}|G(t)|^{2\left\lfloor\frac{\eta \delta T}{4}\right\rfloor-2} \int_{\mathbb{R} \backslash\left(\xi_{0}-\delta, \xi_{0}+\delta\right)}|G(t)|^{2} d \mu(t) \\
\leq & w\left(\xi_{0}\right)(1+\epsilon) \int_{\mathbb{R}}|F(t)|^{2} d t+\left(\frac{1}{2}\right)^{2\left\lfloor\frac{\eta \delta T}{4}\right\rfloor-2} \frac{\delta^{2}}{16} \int_{\mathbb{R} \backslash\left(\xi_{0}-\delta, \xi_{0}+\delta\right)} \frac{d \mu(t)}{|t-\xi|^{2}} \\
\leq & w\left(\xi_{0}\right)(1+\epsilon) \frac{\pi}{\tau}+\left(\frac{1}{2}\right)^{2\left\lfloor\frac{\eta \delta T}{4}\right\rfloor-2} \delta_{t \in \mathbb{R} \backslash\left(\xi_{0}-\delta, \xi_{0}+\delta\right)} \frac{t^{2}+1}{|t-\xi|^{2}} .
\end{aligned}
$$

So, for all $T$ large enough (so that $\xi_{0}+a / T \in\left(\xi_{0}-\delta / 2, \xi_{0}+\delta / 2\right)$ ) we have

$$
\frac{T}{K_{T}\left(\xi_{0}+\frac{a}{T}, \xi_{0}+\frac{a}{T}\right)} \leq \pi w\left(\xi_{0}\right)(1+\epsilon) \frac{T}{\tau}+T\left(\frac{1}{2}\right)^{2\left\lfloor\frac{\eta \delta T}{4}\right\rfloor-2} \delta^{2} C(\delta) .
$$

Thus,

$$
\limsup _{T \rightarrow \infty} \frac{T}{K_{T}\left(\xi_{0}+\frac{a}{T}, \xi_{0}+\frac{a}{T}\right)} \leq \pi w\left(\xi_{0}\right) \frac{1+\epsilon}{1-\eta} .
$$

Since $\epsilon$ and $\eta$ were arbitrary (and independent of each other), we finally obtain

$$
\limsup _{T \rightarrow \infty} \frac{T}{K_{T}\left(\xi_{0}+\frac{a}{T}, \xi_{0}+\frac{a}{T}\right)} \leq \pi w\left(\xi_{0}\right) .
$$

To get the opposite inequality we begin similarly but with the roles of $\mu$ and the Lebesgue measure $m$ reversed. Let $\epsilon, \delta, \xi, T, \tau$, and $\eta$ be given as above. Now, we define $F(z)$ to be the minimizer for the measure $\mu$ by

$$
F(z)=\frac{K_{\tau}(z, \xi)}{K_{\tau}(\xi, \xi)}
$$

Clearly, $F(\xi)=1$ and $1 / K_{\tau}(\xi, \xi)=\int_{\mathbb{R}}|F(t)|^{2} d \mu(t)$. Keeping $G(z)$ the same as above, we define $H(z)$ as in (2.1). We have

$$
\begin{aligned}
\frac{\pi}{T}=\frac{1}{S_{T}(\xi, \xi)} \leq & \int_{\mathbb{R}}|H(z)|^{2} d t \\
\leq & \frac{1+\epsilon}{w\left(\xi_{0}\right)} \int_{\xi_{0}-\delta}^{\xi_{0}+\delta}|F(t)|^{2} d \mu(t) \\
& +\sup _{t \notin\left(\xi_{0}-\delta, \xi_{0}+\delta\right)}|F(t)|^{2}\left(\frac{1}{2}\right)^{2\left[\frac{\eta \delta T}{4}\right]-2} \delta^{2} C \sup _{t \notin\left(\xi_{0}-\delta, \xi_{0}+\delta\right)} \frac{t^{2}+1}{|t-\xi|^{2}}
\end{aligned}
$$

Next, we use the regularity of $\mu$ to conclude that for any $\epsilon_{1}>0$ and any $t \in \mathbb{R}$ we have

$$
|F(t)|^{2} \leq K_{\tau}(t, t) \int_{\mathbb{R}}|F(s)|^{2} d \mu(s) \leq \frac{C_{1} e^{\epsilon_{1} \tau}}{K_{\tau}(\xi, \xi)}
$$


Using this inequality we get

$$
\frac{\pi}{T} \leq \frac{1+\epsilon}{w\left(\xi_{0}\right) K_{\tau}(\xi, \xi)}+\frac{C_{1} e^{\epsilon_{1} \tau}}{K_{\tau}(\xi, \xi)}\left(\frac{1}{2}\right)^{2\left\lfloor\frac{\eta \delta T}{4}\right\rfloor-2} \delta^{2} C \sup _{t \notin\left(\xi_{0}-\delta, \xi_{0}+\delta\right)} \frac{t^{2}+1}{|t-\xi|^{2}}
$$

From the last inequality, by choosing $\epsilon_{1}>0$ small enough and $\tau$ (and hence $T$ ) large enough, we obtain after self-evident algebra that

$$
\frac{K_{\tau}(\xi, \xi)}{\tau} \leq \frac{1+\epsilon}{\pi w\left(\xi_{0}\right)} \frac{T}{\tau}+\frac{T}{\pi \tau}\left(\frac{3}{4}\right)^{2\left\lfloor\frac{\eta \delta T}{4}\right\rfloor-2} \delta^{2} C^{\prime}
$$

Finally, using the fact that the dependence of $\tau=\tau(T)$ on $T$ is surjective, we get much as before that

$$
\limsup _{\tau \rightarrow \infty} \frac{K_{\tau}\left(\xi_{0}+\frac{a}{\tau}, \xi_{0}+\frac{a}{\tau}\right)}{\tau}=\frac{1}{\pi w\left(\xi_{0}\right)} .
$$

Next we will establish Lubinsky's inequality [6] in our setting.

Proposition 2.2. Let $\mu$ and $\mu^{*}$ be two Poisson summable Borel measures such that $\mu \leq \mu^{*}$. Assume that for some $T>0, \mathcal{E}_{T}(\mu)$ is a proper subspace of $L^{2}(\mu)$. Let $K_{T}(z, w)$ and $K_{T}^{*}(z, w)$ be the reproducing kernels in $\mathcal{E}_{T}(\mu)$ and $\mathcal{E}_{T}\left(\mu^{*}\right)$. Then for all $x, y \in \mathbb{R}$ the following inequality holds:

$$
\left|K_{T}(x, y)-K_{T}^{*}(x, y)\right| \leq \sqrt{K_{T}(y, y)\left(K_{T}(x, x)-K_{T}^{*}(x, x)\right)} .
$$

Proof. Notice first that the condition on the measures implies that $\mathcal{E}_{T}\left(\mu^{*}\right) \subset \mathcal{E}_{T}(\mu)$. Moreover, $\mathcal{E}_{T}(\mu)$ and $\mathcal{E}_{T}\left(\mu^{*}\right)$ are both de Branges spaces consisting of entire functions of the Cartwright class and of type no greater than $T$.

For any function $F(z) \in \mathcal{E}_{T}(\mu)$ we have

$$
|F(y)| \leq \sqrt{K_{T}(y, y)}\|F\|_{L^{2}(\mu)} .
$$

To obtain the desired inequality we apply (2.2) to the function $F(z)=K_{T}(x, \bar{z})-$ $K_{T}^{*}(x, \bar{z}) \in \mathcal{E}_{T}(\mu)$ and estimate $\left\|K_{T}(x, t)-K_{T}^{*}(x, t)\right\|_{L^{2}(\mu(t))}$. For this, first notice that

$$
\begin{aligned}
& \left\|K_{T}(x, t)-K_{T}^{*}(x, t)\right\|_{L^{2}(\mu(t))}^{2} \\
& \quad=\left\|K_{T}(x, t)\right\|_{L^{2}(\mu(t))}^{2}-2 \Re\left\langle K_{T}(x, t) \mid K_{T}^{*}(x, t)\right\rangle_{L^{2}(\mu(t))}+\left\|K_{T}^{*}(x, t)\right\|_{L^{2}(\mu(t))}^{2} .
\end{aligned}
$$

Then, using the facts that $K_{T}(x, \bar{z}) \in \mathcal{E}_{T}(\mu)$ as a function of $z$ for the second term and that $\mu \leq \mu^{*}$ for the last term, we obtain

$$
\begin{aligned}
\left\|K_{T}(x, t)-K_{T}^{*}(x, t)\right\|_{L^{2}(\mu(t))}^{2} & \leq K_{T}(x, x)-2 K_{T}^{*}(x, x)+K_{T}^{*}(x, x) \\
& =K_{T}(x, x)-K_{T}^{*}(x, x) .
\end{aligned}
$$

Proof of Theorem 1.2. Define $\nu=\max \left\{\mu, w\left(\xi_{0}\right) m\right\}$. This new measure $\nu$ is a Poisson summable positive Borel measure on $\mathbb{R}$. It is regular since due to $\mu \leq \nu$ we have that $K_{T}^{\nu}(z, w) \leq K_{T}(z, w)$. Here, $K_{T}^{\nu}(z, w)$ and $K_{T}(z, w)$ are the reproducing kernels corresponding to $\nu$ and $\mu$ respectively. In addition, $\nu$ is absolutely continuous in an open set containing $\xi_{0}$ and its the density $v$ is positive and continuous at $\xi_{0}$. Therefore, we can apply the previous proposition and conclude that

$$
\lim _{T \rightarrow \infty} \frac{K_{T}^{\nu}\left(\xi_{0}+\frac{a}{T}, \xi_{0}+\frac{a}{T}\right)}{T}=\frac{1}{\pi v\left(\xi_{0}\right)}=\frac{1}{\pi w\left(\xi_{0}\right)} .
$$


Since $\mu \leq \nu$, we can apply Proposition 2.2 and obtain

$$
\left|K_{T}(x, y)-K_{T}^{\nu}(x, y)\right| \leq \sqrt{K_{T}(y, y)\left(K_{T}(x, x)-K_{T}^{\nu}(x, x)\right)} .
$$

If we set $x=\xi_{0}+a / T$ and $y=\xi_{0}+b / T$ and take the limit as $T \rightarrow \infty$, we get

$$
\lim _{T \rightarrow \infty} \frac{\left|K_{T}\left(\xi_{0}+a / T, \xi_{0}+b / T\right)-K_{T}^{\nu}\left(\xi_{0}+a / T, \xi_{0}+b / T\right)\right|}{T}=0 .
$$

Repeating the same thing for $w\left(\xi_{0}\right) m \leq \nu$ yields

$$
\lim _{T \rightarrow \infty} \frac{\left|S_{T}\left(\xi_{0}+a / T, \xi_{0}+b / T\right) / w\left(\xi_{0}\right)-K_{T}^{\nu}\left(\xi_{0}+a / T, \xi_{0}+b / T\right)\right|}{T}=0 .
$$

Combining the last two limits with the triangle inequality we get

$$
\lim _{T \rightarrow \infty} \frac{K_{T}\left(\xi_{0}+a / T, \xi_{0}+b / T\right)}{T}=\frac{1}{\pi w\left(\xi_{0}\right)} \frac{\sin (a-b)}{a-b} .
$$

Finally, Proposition 2.1 implies the desired limit,

$$
\lim _{T \rightarrow \infty} \frac{K_{T}\left(\xi_{0}+a / T, \xi_{0}+b / T\right)}{K_{T}\left(\xi_{0}, \xi_{0}\right)}=\frac{\sin (a-b)}{a-b} .
$$

\section{REFERENCES}

[1] P. Deift, T. Kriecherbauer, K.T.-R. McLaughlin, S. Venakides, and X. Zhou, Asymptotics for polynomials orthogonal with respect to varying exponential weights, Int. Math. Res. Not. IMRN 1997 (1997), 759-782. MR 1472344 (99g:34038) $\uparrow$

[2] _ Strong asymptotics of orthogonal polynomials with respect to exponential weights, Comm. Pure Appl. Math. 52 (1999), 1491-1552. MR1711036 (2001f:42037) $\uparrow$

[3] P. Deift and X. Zhou, A steepest descent method for oscillatory Riemann-Hilbert problems. Asymptotics for the mKdV equation, Ann. Math. 137 (1993), 295-370. MR.1207209 $(94 \mathrm{~d}: 35143) \uparrow$

[4] M.G. Krein, On a fundamental approximation problem in the theory of extrapolation and filtration of stationary random processes, Dokl. Akad. Nauk SSSR 94 (1954), 13-16. MR.0062980(16:53b) 个

[5] E. Levin and D.S. Lubinsky, Universality limits in the bulk for varying measures, Adv. Math. 219 (2008), 743-779. MR2442052(2010a:60009) $\uparrow$

[6] D.S. Lubinsky, A new approach to universality limits involving orthogonal polynomials, Ann. Math. 170 (2009), 915-939. MR2552113(2011a:42042) $\uparrow$

[7] _ Universality limits in the bulk for arbitrary measures on compact sets, J. Anal. Math. 106 (2008), 373-394. MR2448991 (2009j:30085) $\uparrow$

[8] L. Pastur and M. Scherbina, Universality of the local eigenvalue statistics for a class of unitary invariant random matrix ensembles, J. Stat. Phys. 86 (1997), 109-147. MR.1435193 $(98 \mathrm{~b}: 82037) \uparrow$

[9] B. Simon, Two extensions of Lubinsky's Universality Theorem, J. Anal. Math. 105 (2008), 345-362. MR 2438429 (2010c:42054) $\uparrow$

[10] V. Totik, Universality and fine zero spacing on general sets, Ark. Mat. 47 (2009), 361-391. MR:2529707(2010f:42055) $\uparrow$

School of Mathematics, Georgia Institute of Technology, 686 Cherry Street, Atlanta, Georgia 30332-0160

E-mail address: mishko@math.gatech.edu

Current address: Department of Mathematical Sciences, Clemson University, Clemson, South Carolina 29634

E-mail address: mmitkov@clemson.edu 